\title{
Looking for a needle in a haystack: inference about individual fitness components in a heterogeneous population
}
1. Emmanuelle Cam,
2. Olivier Gimenez,
3. Russell Alpizar-Jara,
4. Lise M. Aubry,
5. Matthieu Authier,
6. Evan G. Cooch,
7. David N. Koons,
8. William A. Link,
9. Jean-Yves Monnat,
10. James D. Nichols,
11. Jay J. Rotella,
12. Jeffrey A. Royle,
13. Roger Pradel

Article first published online: 11 SEP 2012

DOI: 10.1111/j.1600-0706.2012.20532.x

(C) 2012 The Authors

Issue

\section{OIKOS}

\section{Oikos}

Early View (Online Version of Record published before inclusion in an issue) (/journal/10.1111/(ISSN)1600$\underline{0706 / \text { earlyview) }}$

Additional Information

How to Cite 
Cam, E., Gimenez, O., Alpizar-Jara, R., Aubry, L. M., Authier, M., Cooch, E. G., Koons, D. N., Link, W. A., Monnat, J.-Y., Nichols, J. D., Rotella, J. J., Royle, J. A. and Pradel, R. (2012), Looking for a needle in a haystack: inference about individual fitness components in a heterogeneous population. Oikos. doi: 10.1111/j.1600-

0706.2012.20532.x

\section{Author Information}

Centre d'Écologie Évolutive et Fonctionnelle, UMR 51751919 Route de Mende, FR-34293 Montpellier Cedex 5, France.

*E. Cam, Centre d'Écologie Évolutive et Fonctionnelle, UMR 51751919 Route de Mende, FR-34293

Montpellier Cedex 5, France. E-mail: emmanuelle.cam@univ-tlse3.fr

(mailto:emmanuelle.cam@univ\%E2\%80\%90tlse3.fr)

\section{Publication History}

1. Article first published online: 11 SEP 2012

2. Paper manuscript accepted 7 June 2012

- Abstract

- Article (/doi/10.1111/j.1600-0706.2012.20532.x/full)

- References (/doi/10.1111/j.1600-0706.2012.20532.x/references)

- Cited By (/doi/10.1111/j.1600-0706.2012.20532.x/citedby)

View Full Article (HTML) (/doi/10.1111/j.1600-0706.2012.20532.x/full) Get PDF (357K) (/doi/10.1111/j.1600$\underline{0706.2012 .20532 . x / p d f)}$

Studies of wild vertebrates have provided evidence of substantial differences in lifetime reproduction among individuals and the sequences of life history 'states' during life (breeding, nonbreeding, etc.). Such differences may reflect 'fixed' differences in fitness components among individuals determined before, or at the onset of reproductive life. Many retrospective life history studies have translated this idea by assuming a 'latent' unobserved heterogeneity resulting in a fixed hierarchy among individuals in fitness components. Alternatively, fixed differences among individuals are not necessarily needed to account for observed levels of individual heterogeneity in life histories. Individuals with identical fitness traits may stochastically experience different outcomes for breeding and survival through life that lead to a diversity of 'state' sequences with some individuals living longer and being more productive than others, by chance alone. The question is whether individuals differ in their underlying fitness components in ways that cannot be explained by observable 'states' such as age, previous breeding success, etc. Here, we compare statistical models that represent these opposing hypotheses, and mixtures of them, using data from kittiwakes. We constructed models that accounted for observed covariates, individual random effects (unobserved heterogeneity), first-order Markovian transitions between observed states, or combinations of these features. We show that individual sequences of states are better accounted for by models incorporating unobserved heterogeneity than by models including first-order Markov processes alone, or a combination of both. If we had not considered individual heterogeneity, models including Markovian transitions would have been the best performing ones. We also show that inference about age-related changes in fitness components is sensitive to incorporation of underlying individual heterogeneity in models. Our approach provides insight into the sources of individual heterogeneity in life histories, and can be applied to other data sets to examine the ubiquity of our results across the tree of life. 
View Full Article (HTML) (/doi/10.1111/j.1600-0706.2012.20532.x/full) Get PDF (357K) (/doi/10.1111/j.1600$\underline{0706.2012 .20532 . x / \mathrm{pdf})}$

\section{More content like this}

Find more content:

- like this article (/advanced/search/results?articleDoi=10.1111/j.16000706.2012.20532.x\&scope $=$ allContent\&start $=1$ \&resultsPerPage $=20$ )

Find more content written by:

- Emmanuelle Cam (/advanced/search/results?searchRowCriteria[0].queryString="Emmanuelle Cam"\&searchRowCriteria[0]. fieldName=author\&start=1\&resultsPerPage $=20$ )

- Olivier Gimenez (/advanced/search/results?searchRowCriteria[0].queryString="Olivier Gimenez"\&searchRowCriteria[0].fieldName=author\&start=1 \&resultsPerPage=20)

- $\underline{\text { Russell Alpizar-Jara (/advanced/search/results?searchRowCriteria[0].queryString="Russell Alpizar- }}$ Jara"\&searchRowCriteria[0].fieldName=author\&start=1\&resultsPerPage=20)

- Lise M. Aubry (/advanced/search/results?searchRowCriteria[0].queryString="Lise M. Aubry"\&searchRowCriteria[0].fieldName=author\&start=1\&resultsPerPage $=20$ )

- Matthieu Authier (/advanced/search/results?searchRowCriteria[0].queryString="Matthieu Authier"\&searchRowCriteria[0].fieldName=author\&start=1\&resultsPerPage=20)

- Evan G. Cooch (/advanced/search/results?searchRowCriteria[0].queryString="Evan G. Cooch"\&searchRowCriteria[0].fieldName=author\&start=1\&resultsPerPage=20)

- David N. Koons (/advanced/search/results?searchRowCriteria[0].queryString="David N. Koons"\&searchRowCriteria[0].fieldName=author\&start=1\&resultsPerPage=20)

- William A. Link (/advanced/search/results?searchRowCriteria[0].queryString="William A. Link"\&searchRowCriteria[0].fieldName=author\&start=1\&resultsPerPage $=20$ )

- Jean-Yves Monnat (/advanced/search/results?searchRowCriteria[0].queryString="Jean-Yves Monnat"\&searchRowCriteria[0].fieldName=author\&start=1\&resultsPerPage=20)

- James D. Nichols (/advanced/search/results?searchRowCriteria[0].queryString="James D. Nichols"\&searchRowCriteria[0].fieldName=author\&start $=1$ \&resultsPerPage $=20$ )

- $\underline{\text { Jay J. Rotella (/advanced/search/results?searchRowCriteria[0].queryString }=" J a y ~ J . ~}$ Rotella"\&searchRowCriteria[0].fieldName=author\&start=1\&resultsPerPage=20)

- Jeffrey A. Royle (/advanced/search/results?searchRowCriteria[0].queryString="Jeffrey A. $\underline{\text { Royle"\&searchRowCriteria[0].fieldName=author\&start=1\&resultsPerPage }=20 \text { ) }}$

- $\underline{\text { Roger Pradel (/advanced/search/results?searchRowCriteria[0].queryString="Roger }}$ Pradel"\&searchRowCriteria[0].fieldName=author\&start=1\&resultsPerPage=20)

- All Authors (/advanced/search/results?searchRowCriteria[0].queryString="Emmanuelle Cam" "Olivier Gimenez" "Russell Alpizar-Jara" "Lise M. Aubry" "Matthieu Authier" "Evan G. Cooch" "David N. Koons" "William A. Link" "Jean-Yves Monnat" "James D. Nichols" "Jay J. Rotella" "Jeffrey A. Royle" "Roger Pradel"\&searchRowCriteria[0].fieldName=author\&start=1\&resultsPerPage=20) 